\title{
Spin-Orbit Angles as a Probe to Orbital Evolution
}

\author{
A. H. M. J. Triaud ${ }^{1}$, A. Collier Cameron ${ }^{2}$, D. Queloz ${ }^{3}$, D. R.

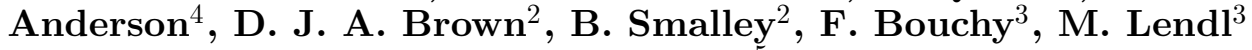 \\ and M. Gillon \\ ${ }^{1}$ Department of Physics, Kavli Institute of Astrophysics \& Space Research, Massachusetts \\ Institute of Technology, Cambridge, MA 02139, USA \\ ${ }^{2}$ SUPA, School of Physics and Astronomy, University of St Andrews, North Haugh, St \\ Andrews, Fife KY16 9SS, UK \\ ${ }^{3}$ Observatoire Astronomique de l'Université de Genève, 51 Chemin des Maillettes, CH-1290 \\ Sauverny, Switzerland \\ ${ }^{4}$ Astrophysics Group, School of Physical and Geographical Sciences, Lennard-Jones Building, \\ Keele University, Staffordshire ST5 5BG, UK \\ ${ }^{5}$ Institut d'Astrophysique et de Geéophysique, Université de Liège, Allée du 6 Août, 17 (Bât. \\ B5C) Sart Tilman, B-4000 Liége, Belgium
}

\begin{abstract}
We observed with HARPS, the Rossiter-McLaughlin effect for 40 of the 75 transiting hot Jupiters discovered in the Southern Hemisphere by WASP. Our observations reveal a wide distribution in orbital inclinations indicative of past dynamical interactions. Our data also demonstrate the important effect produced by tidal interactions in shaping the spin-orbit $(\beta)$ angle distribution. We briefly present and interpret the data we collected in a series of graphs.
\end{abstract}

Keywords. binaries: eclipsing, planetary systems, techniques: radial velocities

The Rossiter-McLaughlin effect is observed using radial velocities and occurs when a planet transits its host star. From its shape we can measure the projected angle onto the sky, called $\beta$ (or sometimes $\lambda$ in literature), between the stellar spin axis and the planet's orbital axis (Gaudi \& Winn, 2007).

Fig. 1a \& b show that the fraction of aligned to misaligned objects is larger when planets orbit stars that have a massive outer convective layer (red triangles) corresponding to $\mathrm{T}_{\text {eff }}<6150 \mathrm{~K}$. Tides are dissipated more effectively when there is an outer convective layer (Zahn 1977). Winn et al. (2010) have postulated that tides have changed the distribution in spin-orbit angles of planets orbiting stars $<6150 \mathrm{~K}$, but left the distribution intact for systems $>6350 \mathrm{~K}$. It is still unclear whether a putative misaligned population of planets orbiting the colder stars could tidally realign or would instead tidally infall into the star, and only leave the mostly aligned population that is observed today.

Fig. 2a only displays planets orbiting the "cold star" sub-sample against a proxy for the strength of tidal interactions: $a / R_{\star}$. We observe that the weaker the tides the more likely misaligned orbits occur. As a recent addition, we point towards WASP-80b, who orbits a K7-M0 star. Although the coldest in our sample, it is observed on an inclined, circular orbit just like hot Jupiters orbiting mid F stars (Triaud et al. 2013). This indicates hot Jupiters may have been more frequently misaligned in the past. No pattern with $a / R_{\star}$ is visible for the sample orbiting hotter stars. This is expected: tides are weakened due to the lack of an outer convective layer. But stars $>1.2 M_{\odot}$ are known to cool as they age on the main sequence. As they cool they develop an outer convective layer. We should therefore expect a change of spin-orbit angle $(\beta)$ distribution, as one planet, originally 

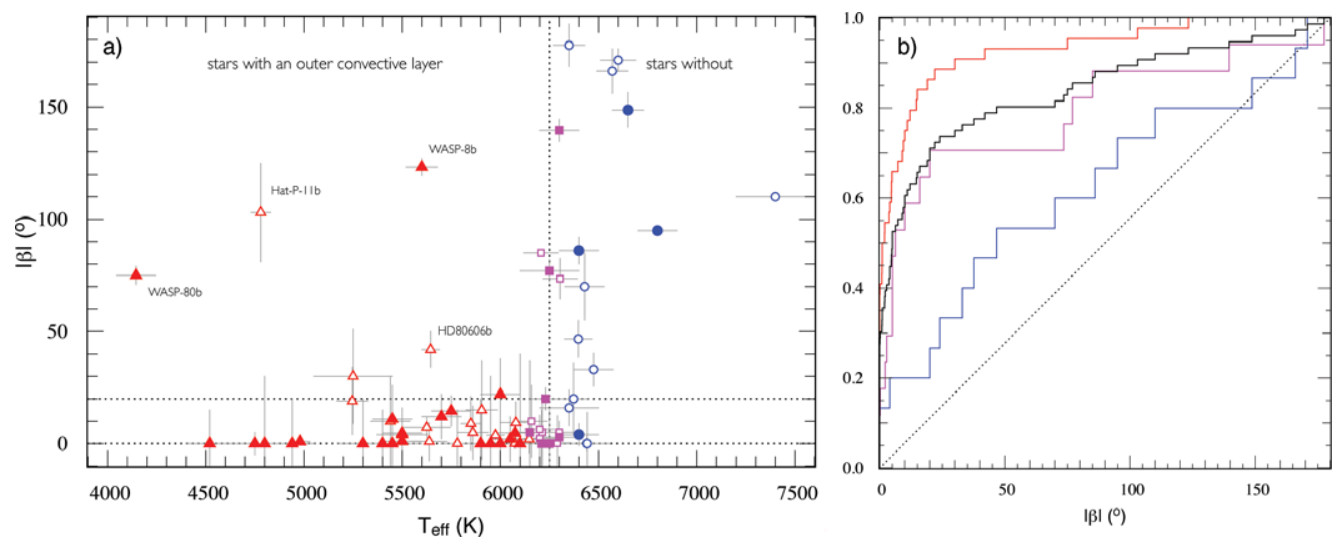

Figure 1. a) updated from Winn et al. (2010). The spin-orbit angle $\beta$ as a function of effective temperature of the host star. Symbols are shaped and colour-coded as a function of $T_{\text {eff }}$. Filled symbols represent our HARPS results. Open diagrams come from other observations efforts. b) show cumulative distribution for each of the different symbol shapes. Top curve is for $T_{\text {eff }}<$ $6150 \mathrm{~K}$ (red triangles), middle for $6150 \leqslant \mathrm{~T}_{\text {eff }}<6350 \mathrm{~K}$ (purple squares), bottom for $\mathrm{T}_{\text {eff }} \geqslant$ $6350 \mathrm{~K}$ (blue discs), with the black cumulative representing the total population. The variety of angles indicate the presence of a process leading some planets onto highly inclined orbits.
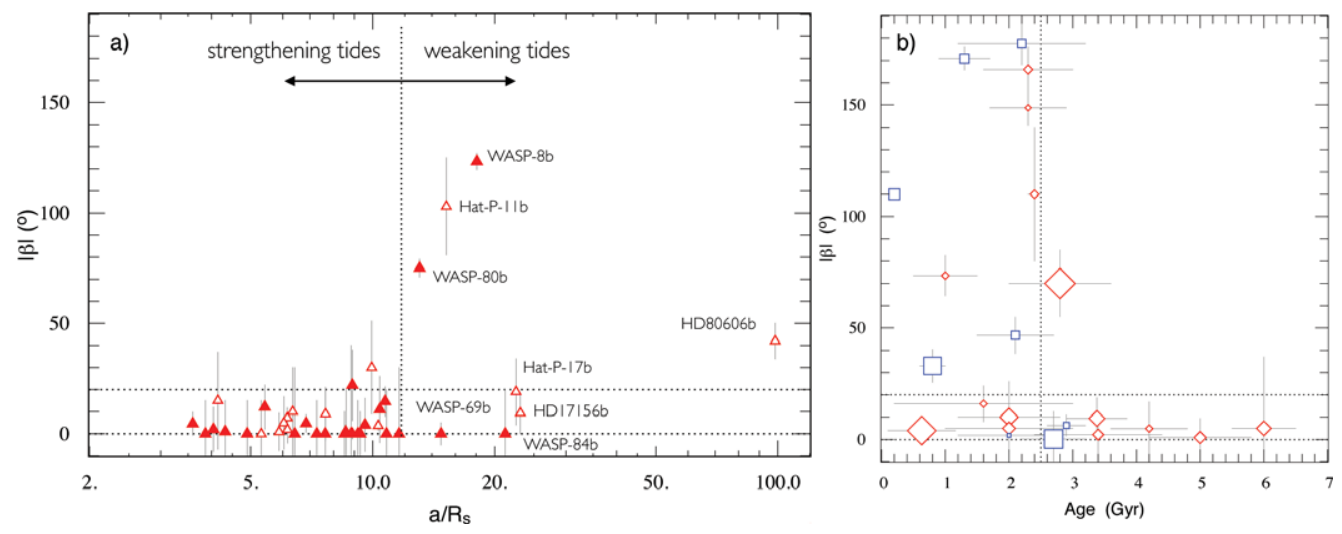

Figure 2. a) updated from Albrecht et al. (2012): spin-orbit angle $\beta$ as a function of $a / R_{\star}$ only selecting object orbiting stars cooler than 6150 (red triangles in Fig. 1a). $a / R_{\star}$ is obtained directly from the transit geometry. It is also a parameter that determines the strength of tides, with the tidal timescale $\tau \propto\left(a / R_{\star}\right)^{-6}$. The larger $a / R_{\star}$ is, the weaker the tides. b) reproduced from Triaud (2011); spin orbit angle $\beta$ as a function of the system's age for primaries $>1.2 M_{\odot}$. Objects older than 2.5-3Gyr are aligned. Ages obtained from the literature.

on the hot side of Fig. 1 will move to the cold side and realign thanks to the development of an outer convective layer on the star. This is what is displayed in Fig. $2 \mathrm{~b}$.

\section{References}

Albrecht, S., Winn, J. N., Johnson, J. A., et al. 2012, ApJ 757, 18

Gaudi, B. S. \& Winn J. N. 2007, ApJ, 655, 550

Triaud, A. H.M. J. 2011, A\&\&A 534, L6

Triaud, A. H. M. J., Anderson, D. R., Doyle, A. P., et al. 2013, A\&A 551, A80

Winn, J. N., Fabrycky, D., Albrecht S. \& Johnson, J. A. 2010, ApJL 718, L145

Zahn, J.-P. 1977, AESA 57, 383 